\title{
Estudo de caso na engenharia de produção: estruturação e recomendações para sua condução
}

\author{
Paulo Augusto Cauchick Miguel \\ POLI-USP
}

\begin{abstract}
Resumo
Uma das preocupações crescentes na engenharia de produção e gestão das operações tanto nos países desenvolvidos quanto no Brasil é com relação às abordagens metodológicas utilizadas no desenvolvimento dos trabalhos de pesquisa, dentre as quais o estudo de caso é uma das mais freqüentemente adotadas. No entanto, a condução adequada de um estudo de caso não é uma tarefa simples e muitas vezes os trabalhos são sujeitos a críticas em função de diversas limitações metodológicas. Nesse sentido, este trabalho propõe uma estrutura para a condução de um estudo de caso[s], bem como sugere um conjunto de recomendações para seu planejamento e condução. Finalmente, alguns pontos importantes são levantados, bem como algumas reflexões e propostas futuras para dar continuidade ao estudo sobre as abordagens metodológicas para a engenharia de produção.
\end{abstract}

Palavras-chave

Engenharia de produção, estudo de caso, metodologia de pesquisa.

\section{Case research in production engineering: structure and recommendations for its conduction}

\begin{abstract}
One of the growing concerns in the production engineering and operations management not only in developed countries but also in Brazil is relatively to the research methodological approaches, among which case research is one of the most frequently adopted. However, the suitable conduction of a case study is not a simple task and, frequently, the research is subjected to criticism due to methodological limitations. In this sense, this work proposes a structure to conduct case studies and suggests a set of recommendations to its research planning and conduction. Finally, some important points are raised as well as some reflections and propositions for future studies in order to keep on with these efforts for continuous improvement in the study of methodological approaches for production engineering.
\end{abstract}

Key words

Production engineering, case study, research methodology. 


\section{INTRODUÇÃO}

A gestão das operações corresponde a um campo do conhecimento que engloba diferentes disciplinas acadêmicas e também seus respectivos campos de aplicação. Um desses campos de aplicação corresponde à engenharia de produção (SLACK et al., 2004). Uma preocupação freqüente na engenharia de produção e gestão das operações nos países desenvolvidos (FILIPPINI, 1997; FILIPPINI; VOSS, 1997) e também no Brasil (BERTO; NAKANO, 1998; 2000) é com relação às abordagens metodológicas utilizadas no desenvolvimento dos trabalhos científicos na área. A importância metodológica de um trabalho pode ser justificada pela necessidade de embasamento científico adequado, geralmente caracterizado pela busca da melhor abordagem de pesquisa a ser utilizada para endereçar as questões da pesquisa, bem como seus respectivos métodos e técnicas para seu planejamento e condução. O resultado é o desenvolvimento de trabalhos melhor estruturados que podem ser replicados e aperfeiçoados por outros pesquisadores visando, acima de tudo, a busca do desenvolvimento da teoria, por meio de sua extensão ou refinamento ou, em última instância, da proposição de novas teorias, contribuindo assim para a geração de conhecimento.

A maior parte da pesquisa conduzida em gestão de operações é baseada em métodos racionalistas de pesquisa, principalmente baseados em análises estatísticas (VOSS et al., 2002). A característica principal da pesquisa racionalista é que o fenômeno existe independentemente do contexto da pesquisa. Entretanto, a gestão de operações é uma disciplina de natureza aplicada, desenvolvida a partir da necessidade de solucionar problemas concretos que surgem nas organizações industriais ou de serviços (FILIPPINI, 1997). Assim, para conduzir as pesquisas nesse campo, diversas alternativas de abordagens metodológicas podem ser utilizadas. Atualmente, as abordagens metodológicas mais utilizadas na engenharia de produção e gestão das operações podem ser categorizadas em: levantamentos tipo survey, modelamento e simulação, pesquisa-ação e estudo de caso.

Dentre essas abordagens, o estudo de caso é uma abordagem extensivamente utilizada, tanto no Brasil quanto nos países desenvolvidos. Analisando os anais do Encontro Nacional de Engenharia de Produção - ENEGEP, um estudo mostrou que o estudo de caso é uma das abordagens mais freqüentes, apesar de limitações no entendimento dos autores dos trabalhos publicados sobre o que realmente significa a condução de um estudo de caso (BERTO; NAKANO, 2000). Dentre os benefícios principais da condução de um estudo dessa natureza estão a possibilidade do desenvolvimento de nova teoria e de aumentar o entendimento sobre eventos reais e contemporâneos, além de que muitos conceitos contemporâneos na gestão de operações e engenharia de produção foram desenvolvidos por meio de estudo de caso (SOUZA, 2005). Entretanto, metodologicamente, a condução adequada de um estudo de caso não é uma tarefa trivial e, freqüentemente, os trabalhos são sujeitos a críticas em função de limitações metodológicas na escolha do(s) caso(s), análise dos dados, e geração de conclusões suportadas pelas evidências. Nesse sentido, este trabalho objetiva fazer algu-

\section{A importância metodológica de um necessidade de embasamento científico adequado, pela busca da melhor abordagem para endereçar as questões da pesquisa.}

mas recomendações para a condução de um estudo de caso, buscando uma maior validade na adoção desse tipo de abordagem metodológica na engenharia de produção. $\mathrm{O}$ trabalho primeiramente fundamenta, em linhas gerais, a abordagem metodológica do presente artigo para, em seguida, enfatizar a metodologia de pesquisa com base na literatura, incluindo uma discussão sobre o estudo de caso como abordagem metodológica na engenharia de produção. Em seguida, descreve resumidamente os tipos de abordagens de pesquisa e também discute a condução do estudo de caso, baseado em etapas definidas, bem como sugere algumas recomendações para seu planejamento e condução. Finalmente, algumas considerações finais são apresentadas, incluindo sugestões para trabalhos futuros.

\section{ABORDAGEM METODOLÓGICA DO PRESENTE TRABALHO}

Metodologicamente, este trabalho é de cunho teóricoconceitual, ou seja, é uma discussão decorrente da análise da literatura, resultando em um levantamento de uma série de pontos relevantes para o planejamento e condução de um estudo de caso(s). Não trata especificamente de uma revisão da literatura, mas apresenta elementos que poderiam levar a essa classificação, pois uma das funções do presente trabalho é identificar, conhecer e acompanhar o desenvolvimento da pesquisa em determinada área do conhecimento. Além disso, busca também identificar algumas perspectivas para pesquisas futuras, o que também é uma função das revisões bibliográficas, segundo Noronha e Ferreira (2000), além de 
buscar exercer o papel de transferir informação do pesquisador para seus pares, conforme atestam algumas pesquisas realizadas sobre o uso das revisões da literatura (SAYERS et al., 1990; BUTKOVICH, 1996).

Utilizando a classificação de Noronha e Ferreira (2000), que categoriza as revisões da literatura segundo seu propósito, abrangência, função e tipo de análise desenvolvida (abordagem), o presente trabalho pode ser classificado como mostra o Quadro 1.

Uma vez estabelecida a linha metodológica deste trabalho, o tópico seguinte discute alguns aspectos sobre metodologia de pesquisa, baseado na literatura.

\section{METODOLOGIA DA PESQUISA CIENTÍFICA}

Uma pesquisa pode ter os seguintes macroobjetivos (SELLTZ et al., 1975): familiarizar com um fenômeno ou conseguir uma nova compreensão sobre ele; apresentar informações sobre uma dada situação, grupo ou entidade; verificar a freqüência com que algo ocorre ou como se liga a outros fenômenos; verificar uma hipótese de relação causal entre variáveis. Geralmente, as pesquisas apresentam características dos quatro tipos anteriores.

Para atender a um ou mais desses macroobjetivos, uma pesquisa desenvolve-se ao longo de um processo que envolve inúmeras fases, desde a adequada formulação do problema até a satisfatória apresentação dos resultados, análise crítica e suas conclusões. O processo de pesquisa é desenvolvido mediante o concurso do conhecimento disponível e a utilização cuidadosa de métodos, técnicas e outros procedimentos científicos. A forma com que o observador interage com o ambiente pesquisado para a detecção dos problemas ou para a proposição de soluções, bem como a maneira como formula as hipóteses, adquire e processa os dados, necessita estar norteado por métodos e técnicas específicos que se adaptem à natureza da pesquisa e à realidade investigada. Pode-se afirmar que não existe um consenso sobre a tipologia das pesquisas, que podem ser classificadas segundo diferentes maneiras, quanto (GODOY, 1995; MAYS; POPE, 1996; MATTAR, 1996):

- à natureza das variáveis pesquisadas - quantitativa ou qualitativa;

- à natureza do relacionamento entre variáveis - caráter descritivo ou causal;

- ao objetivo e ao grau de cristalização do problema - de natureza exploratória ou de natureza conclusiva;

- a intensidade de controle capaz de ser exercida sobre as variáveis em estudo - experimentais em laboratório (variáveis e condições controladas), experimentais de campo (variáveis e condições de difícil controle), ou ex-post facto (isto é, como uma determinada situação ocorreu no passado);

- ao escopo da pesquisa, em termos de profundidade e amplitude - estudo de caso ou levantamentos amostrais tipo survey.

Na gestão de operações e engenharia de produção, a classificação que normalmente se utiliza é com relação ao escopo da pesquisa, considerando também outros tipos de desenvolvimentos, tais como os trabalhos teórico-conceituais, os de modelagem e simulação, dentre outros (FILLIPINI, 1997). Em geral, todos os tipos anteriormente citados estão presentes na metodologia da pesquisa científica em engenharia de produção. Na realidade, esses tipos não são excludentes. É pos-

Quadro 1: Classificação do Presente Trabalho com base em Noronha e Ferreira (2000).

\begin{tabular}{|l|l|l|}
\hline \multicolumn{1}{|c|}{ CLASSIFICAÇÃO } & \multicolumn{1}{|c|}{ TIPO } & \multicolumn{1}{c|}{ RAZÕES PARA ENQUADRAMENTO } \\
\hline Propósito & Analítico & $\begin{array}{l}\text { Por tratar-se de uma revisão sobre um tema específico, agrupamento } \\
\text { parte dos desenvolvimentos ocorridos em uma área de interesse, no } \\
\text { caso, sobre estudo de caso, fornecendo um panorama sobre o tema }\end{array}$ \\
\hline Escopo & Temático & $\begin{array}{l}\text { Em função de que o trabalho é centrado em um recorte específico sobre } \\
\text { o tema estudo de caso }\end{array}$ \\
\hline Função & De atualização & $\begin{array}{l}\text { Por citar estudos publicados recentemente sobre o tema, servindo } \\
\text { tanto para aqueles que se aprofundam no tema como para } \\
\text { pesquisadores iniciantes em um novo projeto usando uma abordagem de } \\
\text { estudo de caso, chamando a atenção para alguns dos trabalhos mais } \\
\text { relevantes nesse tema }\end{array}$ \\
\hline Abordagem & Bibliográfica & $\begin{array}{l}\text { Por ser considerada como uma bibliografia anotada, pois consiste de } \\
\text { um conjunto de fontes sem um aprofundamento em termos de análise } \\
\text { crítica sobre essas fontes, oferecendo uma seleção de trabalhos de } \\
\text { maior interesse no tema estudo de caso }\end{array}$ \\
\hline
\end{tabular}


sível classificar uma pesquisa como de natureza exploratória, sem relação causal entre as variáveis e, portanto, descritiva, utilizando uma abordagem de estudo de caso, com base dados e/ou métodos de natureza qualitativa.

\section{TIPOS DE ABORDAGENS DE PESQUISA}

Segundo algumas fontes (FILIPPINI, 1997; FILIPPINI; VOSS, 1997; BERTO; NAKANO, 2000), as pesquisas mais comuns em engenharia de produção e gestão das operações envolvem os tipos principais resumidos a seguir.

\section{Desenvolvimento teórico-conceitual}

Apesar de os desenvolvimentos teóricos poderem advir de discussões conceituais da literatura ou de revisões bibliográficas (BERTO; NAKANO, 2000), seu escopo principal envolve, sobretudo, modelagens conceituais que resultam em novas teorias. Para um maior entendimento sobre modelamento conceitual, Whetten (1989) relata os ingredientes necessários para uma contribuição teórica, discutindo os elementos essenciais para uma contribuição efetiva à teoria em dada área do conhecimento. Alguns autores apresentam, em essência, o que constitui uma teoria (WACKER, 1998; WHETTEN, 1989) e suas métricas (WACKER, 2004), enquanto que outros a relacionam com o nível empírico (e.g. LEWIS, 1998; EISENHARDT, 1989).

\section{Estudo de caso}

O estudo de caso é um estudo de natureza empírica que investiga um determinado fenômeno, geralmente contemporâneo, dentro de um contexto real de vida, quando as fronteiras entre o fenômeno e o contexto em que ele se insere não são claramente definidas. Trata-se de uma análise aprofundada de um ou mais objetos (casos), para que permita o seu amplo e detalhado conhecimento (GIL, 1996; BERTO; NAKANO, 2000). Seu objetivo é aprofundar o conhecimento acerca de um problema não suficientemente definido (MATTAR, 1996), visando estimular a compreensão, sugerir hipóteses e questões ou desenvolver a teoria. Os estudos de casos podem ser classificados segundo (YIN, 2001; VOSS et al., 2002): seu conteúdo e objetivo final (exploratórios, explanatórios, ou descritivos) ou quantidade de casos (caso único - holístico ou incorporado ou casos múltiplos - também categorizados em holísticos ou incorporados). A principal tendência em todos os tipos de estudo de caso, é que estes tentam esclarecer o motivo pelo qual uma decisão ou um conjunto de decisões foram tomadas, como foram implementadas e com quais resultados alcançados (YIN, 2001).

\section{Levantamentos tipo survey}

Uma survey compreende um levantamento de dados em uma amostra significativa acerca de um problema a ser estuda- do para, em seguida, mediante análise quantitativa, obteremse as conclusões correspondentes aos dados coletados (GIL, 1996). Os levantamentos tipo survey têm como objetivos contribuir para o conhecimento em uma área particular de interesse por meio da coleta de informações sobre indivíduos ou sobre os ambientes desses indivíduos (FORZA, 2002). Ainda segundo o autor anterior, as surveys podem ser exploratórias (adquirir um "insight" inicial sobre um tema e fornecer base para uma survey mais detalhada), confirmatórias (teste de teorias ou explanatórias), ou descritivas (entendimento da relevância de certo fenômeno e descrição da distribuição do fenômeno na população, com o objetivo de fornecer subsídios para construção de teorias ou seu refinamento). Em geral, assume-se como um levantamento do tipo survey, um universo de dezenas, centenas ou milhares de elementos, predominantemente os dois últimos grupos.

\section{Modelamento e Simulação}

$\mathrm{O}$ modelamento ou modelagem compreende o uso de técnicas matemáticas para descrever o funcionamento de um sistema ou parte de um sistema produtivo (BERTO; NAKANO, 2000). Uma complementação é o uso de simulação, que consiste no uso de técnicas computacionais para simular a operação de sistemas produtivos, baseado em um conjunto de variáveis em dado domínio, de forma a investigar a relação causal e quantitativa entre essas variáveis (BERTRAND; FRANSOO, 2002).

\section{Pesquisa-ação}

A pesquisa-ação é um tipo de pesquisa com base empírica que é concebida e realizada em estreita associação com uma ação ou com a resolução de um problema coletivo e na qual os pesquisadores e participantes representativos da situação ou do problema estão envolvidos de modo cooperativo ou participativo (THIOLLENT, 1997). As dez características principais da pesquisa-ação são (COUGHLAN; COGHLAN, 2002): o pesquisador toma ação (não é mero observador); envolve dois objetivos: solucionar um problema e contribuir para a ciência; é interativa (cooperação e interatividade entre os envolvidos); objetiva desenvolver um entendimento holístico; é fundamentalmente relacionada à mudança; requer um entendimento da estrutura étnica (valores e normas); pode incluir todos os tipos de métodos de coleta de dados (técnicas quantitativas e qualitativas); requer um vasto pré-entendimento (do ambiente organizacional, condições, estrutura e dinâmica das operações); deve ser conduzida em tempo real (um estudo de caso "vivo"); requer critérios próprios de qualidade para sua avaliação.

\section{Pesquisa bibliográfica/revisão da literatura}

As revisões da literatura apresentam-se como uma atividade importante para identificar, conhecer e acompanhar 
o desenvolvimento da pesquisa em determinada área do conhecimento (NORONHA; FERREIRA, 2000), além de permitir a cobertura de uma gama de fenômenos geralmente mais ampla do que aquela que poderia ser pesquisada diretamente (GIL, 1996). Além disso, as revisões permitem a identificação de perspectivas para pesquisas futuras, contribuindo com sugestões de idéias para o desenvolvimento de novos projetos de pesquisa (NORONHA; FERREIRA, 2000). De acordo com esses autores, as revisões podem ser classificadas segundo seu propósito (analítica ou de base), abrangência (temporal ou temática), função (histórica ou de atualização) e tipo de análise desenvolvida (bibliográficas ou críticas).

\section{Pesquisas experimentais}

As pesquisas experimentais tratam de um estudo sobre a relação causal entre duas ou mais variáveis de um sistema sob condições controladas pelo pesquisador, geralmente conduzidas em laboratórios. No entanto, Andrade (2002) destaca que a pesquisa de laboratório não é sinônimo de pesquisa experimental, ainda que a maioria das pesquisas de laboratório sejam experimentais. Nesse tipo de pesquisa, em geral, o pesquisador manipula e controla as variáveis e observa as variações que tal manipulação e controle produzem sobre o fenômeno em estudo.

O Quadro 2 apresenta uma série de fontes bibliográficas de acordo com os tipos anteriormente apresentados. É importante mencionar que os livros e artigos que constam no referido quadro não esgotam as possibilidades existentes mas servem apenas como uma referência inicial no estudo sobre metodologia de pesquisa. Além disso, o quadro inclui tanto trabalhos de cunho teórico, ou seja, que estabelecem as bases para condução dos estudos quanto alguns trabalhos de aplicação (denotados por “*”).

Os tipos de pesquisa mostrados no Quadro 2 são utilizados, em maior ou menor grau, na engenharia de produção, mas o estudo de caso é um dos mais adotados e sua estruturação é destacada no tópico a seguir.

\section{ESTRUTURACÃO DO ESTUDO DE CASO}

Fazendo uma analogia com a literatura sobre planejamento estratégico, uma abordagem metodológica adequada compreende diferentes níveis de abrangência e profundidade. Assim, pode-se considerar que algumas decisões metodológicas são de ordem estratégica (decisões relativas à escolha da abordagem mais adequada ao endereçamento da questão de pesquisa), enquanto que outras são de nível tático ou operacional (decisões relativas aos procedimentos de condução da pesquisa). Esses dois níveis são discutidos a seguir.

\section{Nível Estratégico - Abordagem Metodológica}

Um dos problemas com que o pesquisador se depara inicialmente é relativo à escolha da abordagem metodológica da pesquisa. Existe uma grande diversidade de abordagens advindas das ciências exatas ou humanas considerando uma infinidade de objetos de análise, que podem ter vários direcionadores: uma escolha prévia com base na literatura ou em um dado autor específico (como no caso da obra de YIN,

\section{Quadro 2: Fontes Bibliográficas e Tipos de Pesquisas Usados na Engenharia de Produção.}

\begin{tabular}{|l|l|}
\hline \multicolumn{1}{|c|}{$\begin{array}{c}\text { TIPO DE } \\
\text { PESQUISA }\end{array}$} & \multicolumn{1}{c|}{ REFERÂNCIAS } \\
\hline $\begin{array}{l}\text { Desenvolvimento } \\
\text { Teórico-conceitual }\end{array}$ & $\begin{array}{l}\text { Bacharach (1989); Eisenhardt (1989); Weick (1989); Whetten (1989); Locke e Golden-Biddle } \\
\text { (1997); Amundson (1998); Lewis (1980); Melnyk e Handfield (1998); Meredith (1998); Wacker } \\
\text { (1998; 2004); Caldas (2003) }\end{array}$ \\
\hline Estudo de caso & $\begin{array}{l}\text { Eisenhardt (1989); Leonard-Barton (1990); Van de Vem e Huber (1990); McCutcheon e } \\
\text { Meredith (1993); Jayanti e Sinha (1998); Lewis (1998), Hill et al. (1999); Ahltrom e Karlsson } \\
\text { (2000)*; Souza e Voss (2001)*; Yin (2001); Voss et al. (2002); Souza (2003) }\end{array}$ \\
\hline Survey & $\begin{array}{l}\text { Cheng et al. (1994); Collins e Cordon (1997); Adam et al. (1997); Flyn et al. (1997)*; Whybark } \\
\text { (1997)*; Forza (2002); Manel-Samuels (2002)*; Rungtusanatham et al. (2003) }\end{array}$ \\
\hline $\begin{array}{l}\text { Modelamento e } \\
\text { simulação }\end{array}$ & Mitroff et al. (1974); Cheng et al. (1994)*; Bertrand e Fransoo (2002) \\
\hline Pesquisa-ação & $\begin{array}{l}\text { Westbrook (1995); Karlsson e Ahlström (1996)*; Thiollent (1997); Mumford (2001); Coughlan } \\
\text { e Coglan (2002); Zuber-Skerritt e Perry (2002); Coghlan e Coughlan (2003) }\end{array}$ \\
\hline $\begin{array}{l}\text { Pesquisa } \\
\text { bibliográfica }\end{array}$ & Hart (1998; 2001); Noronha e Ferreira (2000); Croom et al. (2000)* \\
\hline
\end{tabular}


2001 para estudo de caso), uma sugestão de um colega, uma recomendação do orientador, ou mesmo por familiaridade ou afinidade com determinado método já empregado no passado. Outros fatores que normalmente são considerados são relativos às contingências típicas de condução da pesquisa, associadas ao objeto de estudo, ao tempo disponível para a finalização da pesquisa, bem como aos recursos financeiros e de tempo para suporte ao trabalho (por exemplo em função dos prazos dados pelas agências de fomento).

$\mathrm{Na}$ verdade, existem premissas e restrições para cada método adotado e estas devem ser levadas em consideração. Entretanto, apesar de os direcionadores apontados anteriormente serem, geralmente, considerados na condução da pesquisa em engenharia de produção, um dos mais importantes é a questão (ou questões) que a pesquisa pretende endereçar. Essa questão é geralmente expressa pelo objetivo do trabalho. O objetivo estabelece a ação (verbo) a ser conduzida e deve, portanto, ser um fator determinante na escolha da abordagem metodológica. Assim, após identificadas as lacunas na área sendo pesquisada, a partir da literatura, e desenvolvida a(s) questão(ões) da pesquisa, o pesquisador deve então estudar as possíveis abordagens a serem utilizadas, selecionando aquela que for mais apropriada, útil, e eficaz para endereçá-la(s) ou, em outras palavras, aquela que deverá atender a problemática estudada no sentido da proposição de soluções. Dessa forma, a adoção de uma abordagem metodológica, como o estudo de caso, deve atender à questão de pesquisa no sentido de proporcionar um caminho para respondê-la.
Para que se busque então atingir os objetivos da pesquisa ou endereçar sua(s) questão(ões), o trabalho deve ser conduzido com o rigor metodológico necessário para que se justifique como uma pesquisa. Assim, faz-se necessário então definir os métodos e técnicas para a coleta dos dados e um planejamento para a condução da pesquisa, discutidos a seguir.

\section{Nível Operacional - A Condução da Investigação}

Uma proposta de conteúdo e seqüência para a condução de um estudo de caso pode ser vista na Figura 1, construída pelo autor com base nos trabalhos de Forza (2002), Croom (2005) e Souza (2005). Na seqüência da figura, cada uma das etapas é então apresentada com maior detalhes.

\section{Definição de uma Estrutura Conceitual-Teórica}

Deve-se primeiramente definir um referencial conceitualteórico para o trabalho, de forma a resultar em um mapeamento da literatura sobre o assunto. Esse mapeamento localiza o tópico de pesquisa no contexto da literatura disponível sobre o tema (CROOM, 2005). O autor complementa que esse mapa indica a abrangência da literatura demonstrando como o tópico em estudo é influenciado pelas fontes bibliográficas existentes. Uma outra função importante nesse mapa é a identificação de trabalhos de cunho teórico ou de caráter empírico. Além disso, a partir da busca bibliográfica e revisão da literatura é possível identificar lacunas onde a pesquisa pode ser justificada (em termos de relevância), bem como possibilita extrair os constructos (constructs:

Figura 1: Condução do Estudo de Caso.

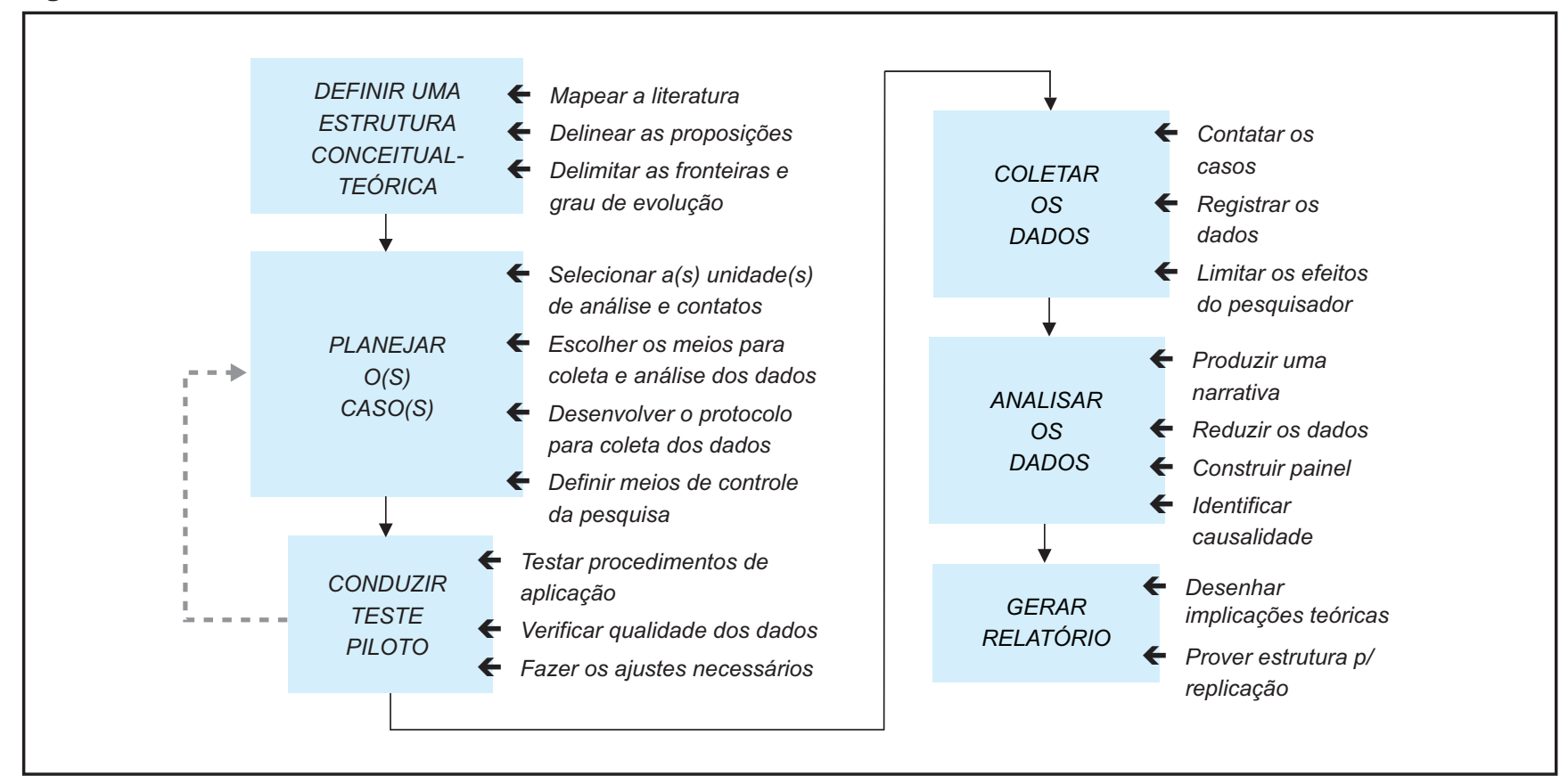


elemento extraído da literatura que representa um conceito a ser verificado, nesse caso empiricamente). A partir desses constructos, as proposições podem ser então estabelecidas (uma ou mais proposições correspondem ao que realmente será verificado, ou seja, é a representação do constructo para fins de mensuração). O referencial teórico também serve para delimitar as fronteiras do que será investigado, proporcionar o suporte teórico para a pesquisa (fundamentos) e também explicitar o grau de evolução (estado da arte) sobre o tema estudado, além de ser um indicativo da familiaridade e conhecimento do pesquisador sobre o assunto. Um trabalho interessante em termos de revisão e classificação da literatura em gestão da cadeia de suprimentos foi feito por Croom et al. (2000). Naquele trabalho é possível verificar uma proposta de classificação da literatura e sua taxinomia, resultando um uma matriz que considera diferentes níveis de análise. No final, o trabalho apresenta algumas implicações para o desenvolvimento da teoria em gestão da cadeia de suprimentos, com base na análise bibliográfica realizada. objetivos da pesquisa, como, por exemplo, se o pesquisador pretende descrever uma mudança em um processo de implantação de dada prática organizacional. Para algumas pesquisas sobre a análise de eficácia implementação de um sistema de produção, por exemplo, pode ser necessária uma análise temporal mais extensa, podendo resultar em uma análise de meses ou anos atrás até o presente. Um exemplo de estudo de caso longitudinal pode ser encontrado em Javanthi e Sinha (1998). Um estudo de caso único permite um maior aprofundamento na investigação e é freqüentemente utilizado em pesquisa longitudinal. Porém, existe uma limitação no grau de generalização (validade externa) uma vez que existe o risco de um julgamento inadequado em função de ser um evento único (SOUZA, 2005). Na adoção de estudo de casos múltiplos, pode-se ter um maior grau de generalização dos resultados, porém espera-se uma profundidade menor na avaliação de cada um dos casos, além de consumir muito mais recursos (YIN, 2001; SOUZA, 2005). Como regra geral, uma quantidade de 4 a 10 casos parece ser suficiente (EISENHARDT, 1989); um excelente exemplo de estudo de caso múltiplo pode ser visto em Souza e Voss (2001) que, inclusive, utilizam métodos de natureza qualitativa e quantitativa na coleta e análise dos dados.

A partir da seleção do(s) caso(s), devese determinar os métodos e técnicas tanto para a coleta quanto para a análise dos dados. Nesse sentido, devem ser empregadas múltiplas fontes de evidência. Usualmente, considera-se entrevistas

\section{Planejamento do(s) Caso(s)}

Uma das primeiras tarefas nesse planejamento é a escolha da(s) unidade(s) de análise, ou seja, do(s) caso(s). Num primeiro momento deve ser determinada a quantidade de casos: único ou múltiplos casos (YIN, 2001), resultando em vantagens e dificuldades em cada um desses tipos. Além disso, o recorte de tempo também é importante, resultando em casos retrospectivos ou longitudinais. Um estudo de caso retrospectivo investiga o passado, coletando dados históricos. Em função da natureza histórica, é difícil determinar relações de causa e efeito, os participantes podem não recordar precisamente os eventos estudados e a análise documental não necessariamente reflete o que realmente ocorreu (SOUZA, 2005). Um estudo de caso longitudinal investiga o presente de certa forma superando as limitações do estudo de caso retrospectivo. No entanto, pode trazer limitações de acesso aos dados e informações, pode resultar em grande consumo de tempo e, não necessariamente, ser conduzido em tempo real (SOUZA, 2005), ou seja, de certa forma pode apresentar alguma retrospectividade. O quanto longitudinal deve ser o estudo de caso vai depender dos (estruturadas, semi-estruturadas ou não estruturadas), análise documental, observações diretas e, embora de forma restrita, pode-se incluir surveys. Quando for o caso, visitas ao "chão de fábrica" também são importantes no sentido de verificar, in loco e/ou in modus operandi, o fenômeno estudado. Eisenhardt (1989) coloca que o uso de múltiplas fontes de dados e a iteração com os constructos desenvolvidos a partir da literatura possibilitam que o pesquisador alcance uma maior validade construtiva da pesquisa. A validade construtiva consiste na extensão pela qual uma observação mede o conceito que se pretende medir (CROOM, 2005). Além disso, o uso de diversas fontes de evidência permite a utilização da técnica de triangulação, que compreende uma iteração entre as diversas fontes de evidência para sustentar os constructos, proposições ou hipóteses, visando analisar a convergência das fontes de evidência. Cabe ainda destacar que as entrevistas devem considerar diferentes indivíduos, em uma perspectiva diversificada em termos de áreas funcionais, níveis hierárquicos, ou quaisquer outras características importantes (como por exemplo o grau de escolaridade ou o sexo). 
Uma vez escolhidas as técnicas para a coleta de dados, um protocolo deve ser desenvolvido. Este não deve se resumir a um roteiro de entrevistas. Além do conjunto de questões a serem usadas, um protocolo deve conter procedimentos e regras gerais da pesquisa para sua condução, indicação da origem das fontes de informação (tipo de fontes, indivíduos, locais, etc.). Dessa forma, um protocolo é mais do que um mero roteiro com perguntas, mas sim um instrumento que melhora a confiabilidade e validade na condução de um estudo de caso. Basicamente, um protocolo deve considerar como partes relevantes (SOUZA, 2005): o contexto (área e local, unidade de análise, questões, procedimentos e fontes de informação), a parte a ser estudada (práticas, unidade de análise, questões, procedimentos e fontes de informação) e meios de controle da pesquisa (variáveis de controle e respectivas questões). Visando alcançar uma maior qualidade na pesquisa, deve-se então definir seus meios de controle, que compreendem uma lista de variáveis que devem ser endereçadas durante a coleta dos dados no sentido das questões que o pesquisador deve ter em mente e que devem ser respondidas sobre cada uma dessas variáveis. Também inclui os procedimentos para serem conduzidos no campo e fontes potenciais de informação para responder às questões. Um protocolo de pesquisa geralmente inclui três partes principais: o contexto da pesquisa, a parte a ser investigada (como por exemplo a utilização de práticas de gestão da qualidade) e as variáveis de controle. A definição dos meios para a análise dos dados é, geralmente, negligenciada na condução de estudo de caso. A análise deve ser previamente planejada e explicitada no trabalho. Apesar de a definição dos métodos a serem adotados também fazer parte dessa etapa, esse detalhamento será feito em etapa mais à frente.

\section{Condução de um Teste Piloto}

Embora não seja uma prática comum em estudo de caso, é sempre importante a condução de um teste piloto pelo pesquisador, antes de partir para a coleta de dados. O objetivo desse teste é verificar os procedimentos de aplicação com base no protocolo, visando seu aprimoramento. A partir dessa aplicação, tem-se também condições de verificar a qualidade dos dados obtidos, visando identificar se eles estão associados aos constructos e, conseqüentemente, se contribuem para o atendimento aos objetivos da pesquisa. A partir do teste fazem-se então as correções e ajustes necessários.

\section{Coleta dos Dados}

Após a realização do teste piloto e possíveis ajustes no protocolo de pesquisa, essa etapa considera a coleta dos dados. Primeiramente, os casos devem ser contatados, considerando os principais informantes que estão cientes da pesquisa. Um contato inicial deve ser um executivo sênior que não somente tenha condições de autorizar a condução da pesquisa, indique quais são os informantes principais que devem ser entrevistados mas também seja capaz de abrir as portas e resolver impasses, caso estes ocorram. Para ter acesso a organização, os contatos pessoais do pesquisador são extremamente úteis, bem como ex-alunos, associações de classe, dentre outros. Deve ficar claro também que a condução da pesquisa deve trazer benefícios mútuos. Esses contatos iniciais podem (na verdade devem) ser feitos com antecedência e, provavelmente, já devem ter sido feitos antes dos dados serem coletados. De qualquer modo, é importante que os informantes tenham clareza do objetivo e importância da pesquisa e o pesquisador assuma o caráter de confidencialidade dos dados coletados. Porém, antes de sair a campo, é importante ter uma estimativa mais clara do tempo a ser despendido e dos recursos a serem consumidos.

\section{estudo de caso é uma espécie de histórico de um fenômeno, extraído de múltiplas fontes de evidências onde qualquer fato} relevante à corrente de eventos que descrevem o fenômeno é um dado potencial para análise.

Após os contatos, os dados devem ser coletados utilizando os instrumentos definidos no planejamento. As habilidades de entrevistas devem ser consideradas, a partir dos seguintes fatores (YIN, 2001): ter capacidade de fazer questões adequadas e interpretar as respostas; ser um bom ouvinte e não trazer nenhum tipo de preconceito; estar muito bem embasado (teoricamente) no tema sendo investigado; ser receptivo e sensível a possíveis evidências contraditórias; ser adaptável e flexível às situações novas e/ou não previstas, considerando-as como oportunidades e não ameaças.

Quanto aos registro dos dados existem várias formas de fazê-lo. Os registros em gravador trazem uma série de vantagens no sentido da melhoria da precisão na análise posterior. Porém, a gravação pode ser intrusiva no sentido de inibir o entrevistado, além da transcrição ser dispendiosa em termos de tempo. Se o uso de gravador não for uma opção desejável, é importante fazer anotações, desenvolvendo os registros das entrevistas. As anotações de campo são extremamente relevantes e todas e quaisquer impressões, descrições do que 
ocorre, e observações devem ser levadas em consideração. Preferencialmente, esses registros devem ser feitos no momento em que os eventos ocorrem. Se isso não for possível, deve ser feito o registro tão logo quanto possível sob o risco de perda de informações importantes. A seqüência dos eventos deve ser planejada (no protocolo) sempre considerando um período de tempo estimado. $\mathrm{O}$ pesquisador também deve buscar convergência e divergência no conjunto de dados e, sempre que necessário, deve também buscar esclarecimento sobre as situações vivenciadas. Se alguma fonte de evidência não foi planejada e foi identificada que é importante, esta deve ser considerada na coleta dos dados.

percam). O mesmo vale para as anotações em papel, que devem ser transferidas para um ou mais arquivos eletrônicos. As anotações e gravações devem ser estruturadas conforme o protocolo de pesquisa. Dados secundários também devem ser utilizados, como por exemplo aqueles relacionados a caracterização do objeto de análise (por exemplo uma empresa). Essa é uma outra (espécie de) transcrição. Outras anotações e impressões, bem como idéias surgidas durante a coleta e insights também devem ser consideradas. Um mecanismo importante para a melhoria na transcrição das narrativas, especialmente se estas não foram gravadas, é o envio do texto para os informantes fazerem uma revisão, dando retorno sobre a exatidão das informações prestadas e também sobre aspectos de confidencialidade que não podem ser divulgados.

No entanto, somente a transcrição e montagem de uma narrativa considerando todas as fontes de evidências não é suficiente para uma análise adequada dos dados. Assim, algumas práticas podem ser utilizadas, como por exemplo a codifica-

Finalmente, deve-se tentar limitar os efeitos do próprio pesquisador, que deve sempre ter em mente que ele(a) é um elemento estranho no contexto analisado; em termos de efeitos do pesquisador no caso, ele(a) pode influenciar os respondentes (SOUZA, 2005). O inverso também é verdadeiro, ou seja, o caso pode influenciar o pesquisador, pois este pode ser induzido pela ingenuidade, ter tendência de concordar com a situação e fazer inferências que, não necessariamente, são decorrentes nas evidências.

A coleta deve ser dada como concluída quando a quantidade de dados e informações reduzir e/ou quando se considera dados suficientes para endereçar a questão da pesquisa. Obviamente, outros aspectos devem ser considerados, como a premência de tempo em função de prazos assumidos. Porém, esse último aspecto não pode influenciar negativamente o rigor metodológico.

\section{Análise dos Dados}

A partir do conjunto de dados coletados, considerando as múltiplas fontes de evidência, o pesquisador deve então produzir uma espécie de narrativa geral do caso. Isso não implica que tudo que foi coletado deverá ser incluído no relatório da pesquisa (seja uma dissertação, tese, relatório de pesquisa ou artigo). Geralmente, será necessário fazer uma redução dos dados (data reduction) de tal forma que seja incluído na análise somente aquilo que é essencial e que tem estreita ligação com os objetivos e constructos da pesquisa. Se houve gravação das entrevistas, essas devem ser transcritas por completo, resultando em dados brutos. A transcrição deve ser feita o mais rapidamente possível para que os detalhes de memória (por exemplo reações, não se ção, que é o primeiro passo para a redução dos dados (SOU$\mathrm{ZA}, 2005$ ). A idéia é marcar as partes da narrativa (palavras, frases ou mesmo parágrafos) com um código que represente categorias previamente definidas. Essas categorias devem corresponder a propriedades teóricas, desdobradas em dimensões associadas à pesquisa. Os códigos são blocos cujo objetivo é resgatar os dados das narrativas e transcrições de forma a associá-los ao que se pretende investigar, seja no âmbito da questão da pesquisa ou dos constructos desenvolvidos a partir da literatura. É importante mencionar que os códigos não respondem a questão da pesquisa, mas são fios condutores para tal, a partir da análise dos dados, descrita a seguir. O Quadro 3 ilustra um exemplo do significado de códigos em uma pesquisa sobre gestão da qualidade. Sempre que uma parte do texto estiver relacionada a um dos códigos (e obviamente com a informação/constructos que se pretende verificar), esta parte é assinalada e colocada em código.

A base da análise é a descrição detalhada do(s) caso(s), pois já nesse estágio possibilita identificar dados e informações relevantes para a pesquisa bem como insights. A seguir, uma espécie de painel demonstrativo de todo o conjunto dos dados deve ser construído. Esse painel é uma representação visual do conjunto de informações para permitir uma visão geral dos dados e ao mesmo tempo detalhada, que permitirá extrair conclusões válidas a partir desses dados. A idéia geral é tornar-se bastante familiar com o conjunto de dados de cada caso. A Figura 2 ilustra um exemplo hipotético de um painel com os dados de dada pesquisa relacionada à gestão da qualidade.

Em suma, o painel com os dados, ilustrado na Figura 2, apresenta um resumo das evidências. Se múltiplos casos 
são empregados, deve-se então construir um painel para cada caso, para, em seguida, fazer uma análise cruzada dos casos identificando convergência e divergência entre as fontes de evidências. É importante mencionar que novas variáveis podem surgir a partir das evidências. Toda esta tarefa pode ser denominada de rede causal, consistindo em displays (painéis) que mostrem o relacionamento entre as variáveis de pesquisa. Assim, num primeiro momento, uma explanação mais geral pode ser elaborada, seguida de uma análise mais detalhada e consistente no sentido de explicar as evidências que podem ser generalizáveis. Essa cadeia de evidências é o que pode levar às conclusões lógicas embasadas nas diversas fontes de dados convergentes. Em paralelo, as conclusões são comparadas com a teoria na tentativa de responder a questão: a teoria pode explicar o fenômeno es- tudado nos contextos diferentes? A partir do entendimento do fenômeno, o pesquisador pode então verificar a literatura existente para apoiar as evidências, empreendendo tentativas de enquadrar os resultados na literatura vigente, o que, não necessariamente, ocorre. A síntese das etapas anteriores em conjunto com os resultados e resposta à questão anterior, por meio das conclusões, é então feita no relatório da pesquisa, descrito a seguir.

\section{Geração do Relatório da Pesquisa}

Todo o conjunto de atividades das etapas anteriores deve então ser sintetizado em um relatório de pesquisa. Esse relatório é o gerador (isto é, não é sinônimo) da monografia (tese ou dissertação) e de artigos (para congressos ou periódicos). Sempre deve ser considerado que os resultados

Quadro 3: Códigos Associados ao Controle da Pesquisa.

\begin{tabular}{|l|l|}
\hline \multicolumn{1}{|c|}{ CÓDIGO } & \multicolumn{1}{c|}{ FORNECE INFORMAÇÃ̃o SOBRE: } \\
\hline DRIVE & Direcionador para adoção de uma prática específica de gestão da qualidade \\
\hline QAWARE & Conscientização da organização sobre as práticas de gestão da qualidade \\
\hline QCULTURE & Extensão pela qual a cultura de qualidade faz parte da organização \\
\hline QEXTIND & Indicadores externos de maturidade de qualidade \\
\hline QHISTORY & Histórico de adoção de práticas de gestão da qualidade na planta \\
\hline
\end{tabular}

Fonte: SOUZA, 2000.

Figura 2: Exemplo llustrativo de Painel dos Dados Coletados.

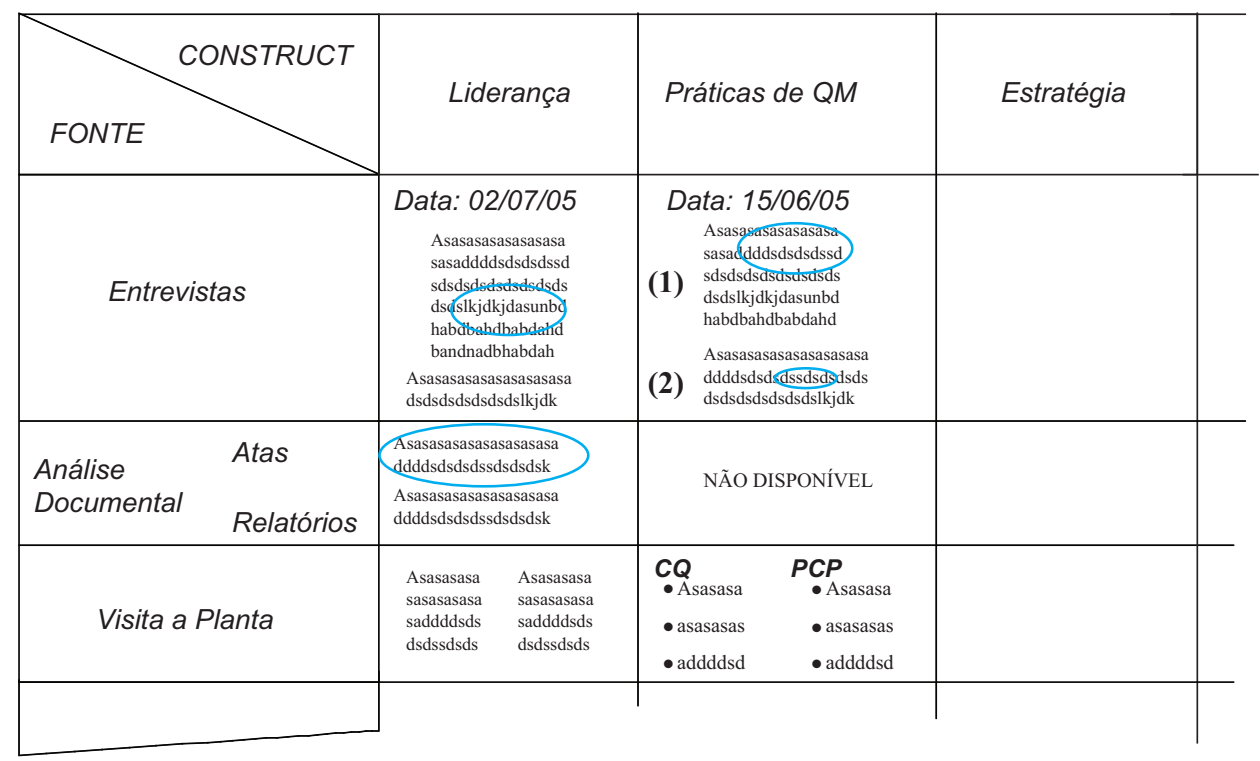


devem estar estreitamente relacionados à teoria, tomando o cuidado para não ajustar a teoria aos resultados e evidências, mas o inverso, ou seja, os resultados e as evidências são o que deve ser associado à teoria, possibilitando, inclusive, a geração de nova teoria.

O estudo de caso deve estar pautado na confiabilidade e validade, que são critérios para julgar a qualidade da pesquisa. A confiabilidade visa demonstrar que as operações de um estudo (como por exemplo os procedimentos para coleta dos dados) podem ser repetidas apresentando os mesmos resultados (YIN, 2001). A validade pode ser subdividida nos diversos tipos mostrados no Quadro 4. Esses os tipos de validade que devem ser levados em consideração não somente no estudo de caso mas também em outros tipos de abordagens metodológicas. Por sua vez, o Quadro 5 mostra alguns dos tipos de validade e da confiabilidade em relação à etapa da pesquisa (Figura 1).

Nesse contexto, este trabalho apresenta na seqüência algumas recomendações para o planejamento e condução do estudo de caso, levando em consideração as macroetapas definidas na Figura 1.

\section{RECOMENDACÕES PARA O ESTUDO DE CASO}

A partir das discussões anteriores, este trabalho sugere uma série de recomendações para o planejamento e condu-

\section{Quadro 4: Tipos de Validade.}

\begin{tabular}{|l|l|}
\hline \multicolumn{1}{|c|}{ VALIDADE } & \multicolumn{1}{c|}{ DEFINIÇÃo } \\
\hline Interna & $\begin{array}{l}\text { Compreende o nível de confiança em relação a causa e efeito entre variáveis. Um exemplo é a } \\
\text { constatação se as conclusões são resultados das evidências }\end{array}$ \\
\hline Externa & $\begin{array}{l}\text { Significa o grau de generalização das conclusões da pesquisa, ou seja, a verificação de quão } \\
\text { aplicáveis são os resultados para outros objetos de análise }\end{array}$ \\
\hline (do) constructo & $\begin{array}{l}\text { Consiste na extensão pela qual uma observação mede o conceito que se pretende medir por } \\
\text { meio do estabelecimento das medidas operacionais corretas em relação a esse conceito }\end{array}$ \\
\hline Descritiva & $\begin{array}{l}\text { É expresso pelo grau pelo qual o relatório da pesquisa é exato, ou seja, representa a situação } \\
\text { pesquisada }\end{array}$ \\
\hline Interpretativa & $\begin{array}{l}\text { Compreende a extensão pela qual a interpretação dada representa o que está sendo } \\
\text { estudado, particularmente no caso na pesquisa empírica }\end{array}$ \\
\hline Teórica & $\begin{array}{l}\text { Consiste no grau pelo qual os dados estão de acordo com a teoria postulada, ou seja, trata- } \\
\text { se da constatação de se a explanação teórica do pesquisador é coerente com os dados } \\
\text { apresentados }\end{array}$ \\
\hline
\end{tabular}

Fonte: Construída a partir de YIN, 2001; CROOM, 2005.

\section{Quadro 5: Validade e Confiabilidade e Etapa da Pesquisa.}

\begin{tabular}{|l|l|l|}
\hline \multicolumn{1}{|c|}{ TESTE } & \multicolumn{1}{|c|}{ ATIVIDADE OPERACIONAL } & \multicolumn{1}{c|}{ ETAPA DA PESQUISA } \\
\hline $\begin{array}{l}\text { Validade do } \\
\text { constructo }\end{array}$ & $\begin{array}{l}\text { Uso de múltiplas fontes de evidências } \\
\text { Estabelecer um encadeamento de evidências } \\
\text { Revisão do relatório pelos respondentes }\end{array}$ & Coleta dos dados \\
\hline Validade interna & $\begin{array}{l}\text { Desenvolver padrão de convergência e de construção da } \\
\text { explanação/narrativa } \\
\text { Fazer análise de séries temporais }\end{array}$ & Análise dos dados \\
\hline Validade externa & Usar a lógica de replicação em múltiplos estudos de caso das & $\begin{array}{l}\text { Planejamento da pesquisa } \\
\text { (casos) }\end{array}$ \\
\hline Confiabilidade & $\begin{array}{l}\text { Usar protocolo de pesquisa no estudo de caso } \\
\text { Desenvolver base de dados para o estudo de caso }\end{array}$ & Coleta de dados \\
\hline
\end{tabular}

Fonte: YIN, 2001. 
ção de um estudo de caso. As seguintes recomendações são de natureza diversa, no sentido de que podem afetar as fases propostas para a condução de um estudo de caso:

- A construção do referencial teórico deve estar estritamente relacionada ao conteúdo do estudo de caso, ou seja, deve identificar as lacunas da pesquisa e prover, quase que naturalmente, a questão que a pesquisa pretende endereçar com a condução do caso. Nesse sentido, existe a necessidade de definição das questões norteadoras da pesquisa (advindas das lacunas identificadas na literatura), bem como relacionadas à necessidade e decisão de conduzir um estudo de caso. Também devem ser considerados as premissas e pressupostos do caso e os mecanismos de iteratividade.

- A definição do tipo de caso (exploratório ou explanatório), em termos de nível de aprofundamento, é um dos primeiros critérios a serem levados em consideração. A utilização relativamente extensiva de casos exploratórios deve considerar que o nível de exploração deve ocorrer quando a teoria não se encontra bem formulada ou se é uma teoria emergente. A não familiaridade do pesquisador com o tema e, conseqüentemente, o enquadramento como exploratório é, no mínimo, questionável sob o ponto de vista metodológico.

- Deve estar bem definida a seleção do(s) caso(s) a ser(em) investigado(s), utilizando-se de critérios robustos que efetivamente justifiquem a escolha feita. Por exemplo, somente a facilidade de acesso ao caso e aos dados é condição necessária mas não suficiente para essa escolha.

- O planejamento do estudo de caso deve ser delineado com cuidado, considerando, além dos aspectos operacionais destacados a seguir, os diversos tipos de validade que ameaçam a caracterização do trabalho de uma pesquisa de cunho científico. Além de prever quais os tipos de validade a que o estudo de caso está sujeito, a descrição do caso deve conter uma análise crítica da qualidade resultante da pesquisa em termos desses diferentes tipos. Infelizmente, essa é uma das maiores negligências na condução de estudo de caso.

- Uma infinidade de fatores devem ser considerados na operacionalização do estudo de caso. Cabe destacar primeiramente a necessidade do uso de múltiplas fontes de evidências e do uso dessas fontes na análise dos dados. Primeiramente, em termos da coleta dos dados, existe a necessidade de uma definição clara de um protocolo de pesquisa. É importante reafirmar que esse protocolo não deve ser limitado somente a uma lista de questões na forma de roteiro de entrevistas. O protocolo deve incluir um guia para a condução do caso, uma definição clara das unidades de análise, como os dados serão coletados e com quem, check lists, itens de controle para a pesquisa, etc. Se a técnica de entrevista é empregada (como ocorre na maioria das vezes), o preparo do entrevistador e sua imparcialidade são aspectos fundamentais para o êxito na coleta dos dados que, como já citado, não deve se limitar a somente essa fonte de evidência (entrevista).

- Não somente a coleta de dados deve ser apresentada, mas também como os dados coletados serão analisados. Devem ser estabelecidos meios apropriados para a análise dos dados, tais como a identificação de padrões nos dados, convergência e divergência, cruzamento de informações (particularmente no uso de múltiplos casos), dentre outros. A análise dos dados deve ser suficientemente robusta para possibilitar uma ligação eficaz com a teoria vigente, levando a sólidas conclusões.

- Considerando as observações anteriores, o caso deve ser robusto o suficiente para que se possa extrair conclusões. Estas devem ser sustentadas com base nas evidências coletadas e na análise dos dados, cujo objetivo final é a contribuição à teoria.

- Finalmente, o objetivo maior da condução de um estudo de caso é a contribuição para a teoria vigente, seja no sentido da proposição de uma nova teoria, extensão da teoria vigente ou de seu refinamento. Não tem sentido a condução do caso per se. $\mathrm{O}$ vínculo com a teoria pode ser obtido considerando as recomendações anteriores. Devese também levar em conta que a abordagem de estudo de caso é limitada em relação a teste de teoria vigente.

Finalmente, considera-se que as recomendações anteriores estejam dentro da contribuição deste trabalho. Espera-se, portanto, que a observação a essas recomendações contribua para colocar o estudo de caso, uma abordagem metodológica extremamente relevante na engenharia de produção no País, em um patamar mais elevado, em condições de equiparar-se ao nível internacional.

\section{CONSIDERACÕES FINAIS}

Primeiramente, é necessário destacar que este trabalho não é conclusivo, no sentido de que deve ter continuidade, visando complementar outras lacunas existentes que, neste momento, não foi possível preencher por completo. Considera-se que a comunidade da engenharia de produção necessita de mobilizar esforços voltados para o estudo metodológico. Se em um passado recente nem ao menos essa discussão estava presente na agenda, hoje em dia existe uma preocupação relativamente generalizada quanto a isso. Os desafios são direcionados a uma busca de que a pesquisa conduzida seja essencialmente científica, embora possam existir divergências na comunidade da engenharia de produção quanto a essa necessidade. Em essência, os trabalhos 
devem apresentar uma coerência e alinhamento nas suas partes principais: referencial teórico, proposições de objetivos, desenvolvimento da proposta (para atingir os objetivos) e conclusões sustentáveis e vinculadas à contribuição para a teoria. Nesse sentido, entende-se que a caracterização da pesquisa, bem como os métodos e técnicas empregados possibilitam alcançar essa coerência e venham a prover maiores condições de explicar os fenômenos estudados.

Especificamente para a engenharia de produção, é necessário mobilizar os esforços da comunidade para a produção de textos que venham a acrescentar e a enriquecer as expe- riências existentes, considerando as particularidades de cada área. Necessita-se, em um primeiro momento, produzir textos didáticos específicos sobre metodologia de pesquisa nos programas de pós-graduação para, em seguida, compará-los e discuti-los à luz de uma maior rigorosidade no desenvolvimento das teses e dissertações. Além disso, é preciso desenvolver uma postura mais crítica da produção científica da engenharia de produção no País, com base no referencial internacional. Nesse sentido, outros trabalhos que analisem as publicações nos periódicos mais importantes da área são relevantes, sendo este um dos trabalhos futuros que o autor pretende desenvolver.

\section{Artigo recebido em 28/06/2006 Aprovado para publicação em 23/02/2007}

\section{- Referências}

ADAM JR., E. et al. An International Study of Quality Improvement Approach and Firm Performance. International Journal of Operations and Production Management, $\mathrm{v}$. 17 , n. 9 , p. $842-873,1997$.

ÅHLDTRÖM, P. ; KARLSSON, C. Sequences of Manufacturing Improvement Initiatives. The Case of Delaying. International Journal of Operations and Production Management, v. 20, n. 11, p. 1259-1277, 2000

AMUNDSON, S. D. Relationships between Theory-driven Empirical Research in Operations Management and Other Disciplines. Journal of Operations Management, v. 16, p. 341-359, 1998.

ANDRADE, M. M. Como Preparar Trabalhos para Cursos de Pós-graduação: Noções Práticas. São Paulo: Atlas, 2002.

BACHARACH, S. B. Organizationa Theories: Some Criteria for Evaluation. The Academy of Management Review, v. 14 n. 4, p. 496-513, 1989.

BERTO, R.M.v.S. e NAKANO, D.N. Métodos de Pesquisa na Engenharia de Produção. CD ROM do XVIII ENEGEP, Niterói, 1998

BERTO, R.M.v.S., NAKANO, D. N. A Produção Científica nos Anais do Encontro Nacional de Engenharia de Produção: Um Levantamento de Métodos e Tipos de Pesquisa. Produção, v. 9, n. 2, p. 65-76, 2000.

BERTRAND, J. W. M.; FRANSOO, J. C. Modeling and Simulation: Operations Management Research Methodology Using Quantitative Modeling. International Journal of Operations and
Production Management, v. 22, n. 2, p. 241-264.

BUTKOVICH, N. J. Reshelving Study of Review Literature in the Physical Science. Library Resources, v. 40, n. 2, p. 139-144, 1996

CALDAS, M. Contribuição Teórica: Como Assim Cara Pálida? RAE - Revista de Administração de Empresas, v. 43, n. 3, p. 65-68, 2003.

CHENG, C. H.; FEIRING, B. R.; CHENG, T. C. E. The Cutting Stock Problem: A Survey. International Journal of Production Economics, v. 36, p. 291-305, 1994.

COGHLAN, D.; COUGHLAN, P. Acquiring the Capacity for Operational Improvement: an Action Research Opportunity. Human Resource Planning, v. 26, n. 2, p. 30-38, 2003.

COLlinS, R.; CORDON, C. Survey Methodology Issues in Manufacturing Strategy and Practice Research. International Journal of Operations and Production Management, v. 17, n. 7; p. 697-706, 1997.

COUGHLAN, P.; COGHLAN, D. Action Research for Operation Management. International Journal of Operations and Production Management, v. 22, n. 2, p. 220-240, 2002.

CROOM, S. Topic Issues and Methodological Concerns for Operations Management Research. EDEN Doctoral Seminar on Research Methodology in Operations Management, Brussels, Belgium, 31st Jan.-4th Feb, 2005.
CROOM, S. R.; ROMANO P.; GIANNAKIS, M. Supply Chain Management: A Literature Review and Taxonomy. European Journa of Purchasing and Supply Management, v. 6, n. 1, p. 67-83, 2000.

EISENHARDT, K. M. Building Theorie from Case Study Research. Academy of Management Review, v. 14 , n. 4, p. 532 $550,1989$.

FILIPPINI, R. Operations Management Research: Some Reflections on Evolution, Models and Empirical Studies in OM. International Journal of Operations and Production Management, v. 17, n. 7, p. 655-670, 1997.

FILIPPINI, R. e VOSS, C. Editorial. International Journal of Operations and Production Management, v. 17, n. 7, p. 653-654, 1997

FORZA, C. Survey Research in Operations Management: a Processbased Perspective. International Journal of Operations \& Production Management, v. 22 , n. 2 , p. $152-194,2002$

FLYN, B.; SCHOEDER, R. G.; FLYN, E. J.; SAKAKIBARA, S.; BATES, K. World-class Manufacturing Project: Overview and Selected Results. International Journal of Operations and Production Management, $\mathrm{v}$. 17, n. 7, p. 671-685, 1997.

GIL, A. C. Como Elaborar Projetos de Pesquisa. São Paulo: Atlas, 1996.

GODOY, A. S. Introdução à Pesquisa Qualitativa e suas Possibilidades. Revista de Administração de Empresas, v. 35, n. 2 , p. 57-63, 1995
HART, C. Doing a Literature Review. London: Sage Publications, 1998.

HART, C. Doing a Literature Search. London: Sage Publications, 2001.

HILL, T.; NICHOLSON, A.; WESTBROOK, R. Closing the Gap: A Polemic on Plant-based Rresearch in Operations Management. International Journal of Operations and Production Management, v. 19, n. 2 , p. 139-156, 1999.

JAYANTI, S.; SINHA, K. Innovation Implementation in High Technology Manufacturing: A Longitudinal Field Study. Journal of Operations Management, v. 16, n. 4, p. 471-494, 1998.

KARLSSON, C.; ÅHLDTRÖM.P. The Difficult Path to Lean Product Development. The Journal of Product Innovation Management, v. 13 , n. 4, p. 283-295, 1996.

LEONARD-BARTON, D. A Dual Methodology for Case Studies: Synergistic Use of Longitudinal Single Site with Replicated Multiple Sites. Organization Science, v. 1, n. 3, p. 248-266, 1990.

LEWIS, M. W. Iterative Triangulation; a Theory Development Process using Existing Case Studies. Journal of Operations Management, v. 16, p. 455469, 1998.

LOCKE, K.; GOLDEN-BIDDLE, K. Constructing Opportunities for Contribution: Structuring Intertextual Coherence and 'Problematizing'in Organizational Studies. Academy of Management Journal, v. 40, n. 5, p. 1023 1062, 1997 
MANNEL-SAMUELS, P. Getting the Truth in Workplace Surveys. Harward Business Review, February, 2002.

MATTAR, F. N. Pesquisa de Marketing: Metodologia e Planejamento. São Paulo: Atlas, 1996.

MAYS, N.; POPE, C. Qualitative Research in Health Care. Londres: BMJ Publishing Group, 1996.

McCUTCHEON, D.; MEREDITH, J. Conducting Case Study Research in Operation Management. Journal of Operations Management, v. 11, p. 239256, 1993.

MEREDITH, J. Building Operations Management Theory through Case and Field Research. Journal of Operations Management, v. 16, p. 441-454, 1998.

MELNYK, S. A.; HANDFIELD, R. B. May You Live in Interesting Times ... The Emergence of Theory-driven Empirical Research. Journal of Operations Management, v. 16, p. 311-319, 1998

MITROFF, I. I.; BETZ, F.; PONDY, L.R.; SAGASTI, F. On Managing Science in the Systems Age: Two Schemas for the Study of Science as a Whole Systems Phenomenon. Interfaces, v. 4, n. 3, p. 46-58.

MUNFORD, E. Advice for an Action Researcher. Information, Technology and People, v. 14, n. 1, p. 12-27, 2001.
NORONHA, D. P.; FERREIRA, S. M. S. P. Revisões da Literatura. In: Campello, B.S., Cendón, B.v. e Kremer, J.M. Fontes de Informação para Pesquisadores e Profissionais. Belo Horizonte: Ed. UFMG, p. $191-198,2000$.

RUNGTUSANATHAM, M. J.; CHOI, T. Y.; HOLLINGWORTH, D. G.; WU, Z.; FORZA, C. Survey Research in Operations Management: Historical Analyses. Journa of Operations Management, v. 21, p. 475488, 2003.

SAYERS. M.; JOICE, J.; BAWDEN, D Retrieval of Biomedical Reviews: a Comparative Evaluation of Online Databases for Reviews of Drug Therapy. Journal of Information Science, v. 16, p. 321-325, 1990.

SELLTZ, C. et al. Métodos de Pesquisa nas Relações Sociais. São Paulo: EDUSP, 1975.

SLACK, N.; LEWIS, M.; BATES, H. The Two Worlds of Operations Management Research and Practice - Can They Meet, Should They Meet? International Journal of Operations and Production Management, v. 24 , n. 4 , p. $372-387,2004$.

SOUZA, R.; VOSS, C. Quality Management Universal or Context Dependent? Production and Operations Management, v. 10 , n. 4 , p. 383-404, 2001.

SOUZA, R. Linking Quality Management to Manufacturing Strategy: An Empirical
Investigation of Customer Focus Practices? Journal of Operations Management, v. 21, n. 1, p. 1-18, 2003.

SOUZA, R. Case Research in Operations Management. EDEN Doctoral Seminar on Research Methodology in Operations Management, Brussels, Belgium, 31st Jan.-4th Feb, 2005.

THIOLLENT, M. Metodologia da Pesquisa Ação. São Paulo: Atlas, 1997.

VAN DE VEM, A.; HUBER, G. Longitudinal Field Research Methods for Studying Process of Organizational Change. Organization Science, v. 1, n. 3, p. 213219, 1990.

VOSS, C. et al. Case Research in Operations Management. International Journal of Operations and Production Management, $\mathrm{v}$. 22, n. 2, p. 195-219, 2002.

WACKER, J. G. A Definition of Theory: Research Guidelines for Different Theorybuilding Research Methods in Operations Management, Journal of Operations Management, v. 16, p. 361-385, 1998.

WACKER, J. G. A Theory of Formal Conceptual Definition: Developing Theory-building Measurement Instruments. Journal of Operations Management, v. 22, p. 629-650, 2004.

WEICK, K. E. Theory Construction as Disciplined Imagination. The Academy of
Management Review, v. 14 , n. 4, p. 516531, 1989.

WESTBROOK, R. Action Research: a New Paradigm for Research in Production and Operations Management. International Journal of Operations and Production Research, v. 15, n. 12, p. 46-58, 1995.

WACKER, J. G. A Definition of Theory Research Guidelines for Different Theorybuilding Research Methods in Operations Management, Journal of Operations Management, v. 16, p. 361-385, 1998.

WHETTEN, D. A. What Constitutes a Theoretical Contribution? Academy of Management Review, v. 14 , n. 4, p. 490495, 1989.

WHYBARK, C. GMRG-Global Manufacturing Research Group Survey Research in Operations Management. International Journal of Operations and Production Management, v. 17, n. 7, p. 686-696, 1997.

ZUBER-SKERRITT, O.; PERRY, C. Action Research within Organizations and University Thesis Writing. The Learning Organization, v. 9, n. 4, p. 171-179, 2002.

YIN, R. K. Estudo de Caso - Planejamento e Método. 2. ed. São Paulo: Bookman, 2001.

\section{- Agradecimentos}

Existem hoje vários pesquisadores que atuam na engenharia de produção e gestão das operações, preocupados com o tema de metodologia de pesquisa. Nesse sentido, o autor deste trabalho agradece esses pesquisadores que, direta ou indiretamente, contribuíram para este texto, seja por meio das discussões sobre o tema, por suas palestras ou pelas oportunidades diversas e convites proporcionados. Dentre estes, podem ser citados: Afonso Fleury, da USP, Alvaro Abackerli, da UNIMEP, Fernando Laurindo, da USP, Henrique Corrêa, da Rollins College nos EUA, José Arantes Salles, da UNIMEP, João Batista Turrioni, da UNIFEI, Lin Chih Cheng, da UFMG, Miguel Caldas, da Universidade de Loyola nos EUA, Roberto Martins, da UFSCar e Rui Souza, da Universidade Católica Portuguesa (Porto). Este trabalho é também resultante da participação do autor no curso Research Methodology in Operations Management, promovido pela European Institute for Advanced Studies in Management, em Bruxelas, na Bélgica, em 2005. Dessa forma, o autor agradece à Fundação Carlos Alberto Vanzolini que o apoiou por meio do fundo de apoio à pesquisa. O autor também é filiado ao Programa de Pós-graduação em Engenharia de Produção da Faculdade de Engenharia (FEAU) da UNIMEP e, portanto, essa instituição também merece seus agradecimentos.

\section{- Sobre o autor}

\section{Paulo Augusto Cauchick Miguel}

Departamento de Engenharia de Produção, Escola Politécnica, USP

End.: Av. Prof. Almeida Prado, trav. 2, no 128 - Cidade Universitária - 05508-900 - São Paulo - SP

E-mail: paulo.miguel@poli.usp.br 